\title{
Estimación de la sustentabilidad de fincas productoras de arveja en el municipio de Ipiales, Nariño-Colombia
}

\section{Estimation of the sustainability of farm producers of vegetables in the municipality of Ipiales, Nariño-Colombia}

\author{
David Álvarez-Sánchez ; Eyder Gómez-López²
}

1Ing. Agrónomo, M.Sc., Ph.D. Universidad de Nariño, Facultad de Ciencias Agrícolas. Pasto - Nariño, Colombia; e-mail: daealvarezsa@udenar.edu.co; (Dhttps://orcid.org/0000-0003-3563-2529

${ }^{2}$ Ing. Agrónomo, M.Sc., Ph.D. Universidad Nacional de Colombia, sede Palmira, Facultad de Ciencias Agropecuarias. Palmira - Valle del Cauca, Colombia; e-mail: eydgomezlo@unal.edu.co; Dhttps://orcid.org/0000-0002-5892-5722

Cómo citar: Álvarez-Sánchez, D.; Gómez-López, E. 2020. Estimación de la sustentabilidad de fincas productoras de arveja en el municipio de Ipiales, Nariño-Colombia. Rev. U.D.C.A Act. \& Div. Cient. 23(1):e1578. http://doi.org/10.31910/rudca.v23.n1.2020.1578

Artículo de acceso abierto publicado por Revista U.D.C.A Actualidad \& Divulgación Científica, bajo una licencia Creative Commons CC BY-NC 4.0

Publicación oficial de la Universidad de Ciencias Aplicadas y Ambientales U.D.C.A, Institución de Educación Superior Acreditada de Alta Calidad por el Ministerio de Educación Nacional.

Recibido: Octubre 29 de 2019

Aceptado Junio 2 de 2020

Editado por: Ingeborg Zenner de Polanía

\section{RESUMEN}

Los agroecosistemas muestran una gran heterogeneidad en cuanto a las prácticas y características, debido a que dependen de las lógicas de la agricultura familiar, convirtiéndose en un recurso potencialmente valioso, cuando se estudia el uso y el soporte del medio productivo. Es por ello, que esta investigación evaluó el sistema de arveja (Pisum sativum L.) en el municipio de Ipiales, a través de una metodología multidimensional, usando 26 indicadores, agrupados en cuatro dimensiones de sustentabilidad agroecológica. La estimación, se realizó sobre 30 fincas, que representaron tres tipos de campesinos, previamente descritos, como C1, C2 y C3; los resultados permitieron identificar los Puntos Críticos de Sustentabilidad (PCS) y el Índice General de Sustentabilidad (IGS) del sistema, estableciendo que las diferentes situaciones sociales, ambientales, económicas y técnicoproductivas determinan la existencia de PCS específicos. El IGS tuvo una calificación de 2,65, para C1; 2,90, para C2 y 2,62, para C3, evidenciando medidas de monitoreo y de manejo, que se deberían discutir en la región, para disminuir las externalidades generadas por el modelo productivo.

Palabras clave: Pisum sativum L.; agroecología; indicadores; agroecosistema.

\section{ABSTRACT}

Agroecosystems present great heterogeneity in terms of practices and characteristics, since they depend on the logic of family farming, which makes them a potentially valuable resource when studying the use and support of the productive environment. For this reason, this research evaluated the pea system (Pisum sativum L.) in the municipality of Ipiales, through a multidimensional methodology that uses 26 indicators grouped into four dimensions 
of agroecological sustainability. The estimation was made on 30 farms that represented three types of farmers previously described as C1, C2 and C3; the results allowed identifying the Critical Points of Sustainability (CPS) and the General Index of Sustainability (GIS) of the system. It was established that the different social, environmental, economic and technical-productive situations determine the existence of specific CPS, the GIS had a rating of 2.65 for $\mathrm{C} 1,2.90$ for $\mathrm{C} 2$ and 2.62 for $\mathrm{C} 3$, this evidenced monitoring and management measures that should be discussed in the region to reduce the externalities generated by the production model.

Keywords: Pisum sativum L.; agroecology; indicators; agroecosystem.

\section{INTRODUCCIÓN}

La manipulación de la naturaleza, con el fin de establecer una producción agrícola intensiva, ha simplificado los agroecosistemas hasta el punto que son estructural y funcionalmente muy diferentes a los ecosistemas naturales (Nicholls et al. 2015). En ellos, las tecnologías empleadas usualmente, aumentan la capacidad de carga por encima de las posibilidades, elevando, a su vez, la necesidad metabólica en el uso de energía y de materia, para obtener los rendimientos esperados (Blandi et al. 2015; Paleologos et al. 2017).

Esta forma de producción constituye la estrategia de organización socioeconómica vigente, de crecimiento y de acumulación de capital, que ha tenido un relativo éxito, pero que, paralelamente, ha generado externalidades negativas, como lo es la contaminación ambiental, la degradación de recursos naturales, el incremento de limitantes en la producción y la concentración de la riqueza, en especial, en los países subdesarrollados (Vélez, 2015).

Bajo este panorama, es inconsecuente estudiar la complejidad agraria con el mismo enfoque, con el cual, se han generado gran parte de los problemas actuales, a lo que la agroecología se ha propuesto como un nuevo paradigma, capaz de integrar, aprender y aplicar los conocimientos producidos en diferentes disciplinas científicas con los saberes y experiencias de los campesinos, de forma holística y sistémica, brindando fundamentos para incrementar la sustentabilidad, como cualidad emergente, a distintas escalas.

Es por ello, que diversos estudios agroecológicos han centrado sus esfuerzos en la evaluación de la sustentabilidad, para evaluar el sistema finca y abordar las problemáticas rurales, generando, desde la autogestión de las comunidades y las redes de cooperación, un desarrollo con responsabilidad intergeneracional, entendiendo el sistema como la conjunción de atributos sociales, ambientales, económicos y técnico-productivos.

Por lo anterior, la presente investigación buscó evaluar tres tipos de fincas dedicadas al cultivo de arveja, previamente caracterizadas y tipificadas, en el municipio de Ipiales, departamento de Nariño (Colombia), por Álvarez et al. (2019), dado que en este municipio, se concentra la principal zona productora del país, con 3,070ha, de acuerdo con las estadísticas oficiales (MADR, 2018), resultado de la expansión que ha tenido este cultivo, a partir de la adopción de variedades mejoradas y tecnología de producción intensiva desde finales de los años noventa, con distintas características de presión sobre el medio.

Ampliando el argumento sobre desarrollo económico a partir del cultivo de arveja, como normalmente se discute, en este estudio, se plantea la inquietud sobre la responsabilidad social, la conservación del ambiente, el suelo y la biodiversidad. De incalculable importancia, si se tiene en cuenta que, aproximadamente, 4.386 familias dependen de la producción de arveja en Ipiales y unas 20.140, en el departamento de Nariño (MADR, 2018).

Para esto, se consideró los trabajos desarrollados por Sarandón \& Flores (2009), quienes integran al concepto de sustentabilidad, la "capacidad del sistema ecológico para proveer de bienes y servicios que satisfagan las necesidades humanas actuales sin comprometer su aprovisionamiento futuro", los requisitos agroecológicos, que determinan un sistema sustentable cuando este es suficientemente productivo, económicamente viable, ecológicamente adecuado y cultural y socialmente aceptado.

\section{MATERIALES Y MÉTODOS}

Localización. La investigación, se desarrolló en 30 fincas dedicadas a la producción de arveja, en una zona agrícola, caracterizada como Bosque Seco Montano Bajo (Bs-MB), con temperatura promedio de $11,9^{\circ} \mathrm{C}$, precipitación media anual de $1.110 \mathrm{~mm}$ y humedad relativa de 81 a 84\%. El sistema de manejo agrotecnológico, en todos los casos, fue agricultura convencional.

La selección de las fincas, se basó en una tipificación previa realizada por Álvarez et al. (2019), quien definió tres clústeres para el sistema productivo de arveja en Nariño; las principales características de los grupos, se resumen así:

C1 = tamaño de finca entre 1 y 2 ha; área dedicada al cultivo de arveja de 1 a 5 ha, debido a la práctica de arrendamiento de lotes; topografía ondulada a quebrada; costo de producción alto; uso de maquinaria agrícola; intensificación productiva, rendimiento alto; contratación de jornales.

C2 = tamaño de finca entre 0,5 y 1 ha; área dedicada al cultivo de arveja de 0,3 a 0,6 ha; topografía plana a ondulada; rendimiento intermedio; contratación jornales o trabajo familiar; estrategia de asociatividad.

C3 = tamaño de finca entre 0,5 y 1ha; área dedicada al cultivo de arveja de 0,5 a 1 ha; topografía quebrada; rendimiento bajo; trabajo familiar; estrategia de asociatividad; ingreso económico bajo.

A partir de recorridos por el municipio fueron seleccionados diez predios por cada clúster, en los cuales, se sembró el cultivo en la misma fecha para garantizar mayor uniformidad en las evaluaciones.

Marco de evaluación de sustentabilidad. Se aplicó la metodología propuesta por Sarandón \& Flores (2009), a través de un proceso 
participativo; para ello, se abordó la dimensión social, ambiental, económica y técnico-productiva, a través de 11 macroindicadores y 26 indicadores.

La estimación, se llevó a cabo en dos etapas. En el cuadro 1, se describen los indicadores usados, parametrizados a partir de evaluaciones exploratorias en el municipio y diagnóstico con campesinos e ingenieros agrónomos, tomando una calificación entre 1 a 4, siendo 4 el valor más alto y 1 el valor más bajo. Como referencia, se usó el valor intermedio de la escala, por debajo del cual, se consideró que no se estaría cumpliendo con los requisitos de sustentabilidad en la finca (Sarandón \& Flores, 2009; Blandi et al. 2015).

Cuadro 1. Descripción de los indicadores y las dimensiones usadas para estimar la sustentabilidad de fincas productoras de arveja en Ipiales-Nariño.

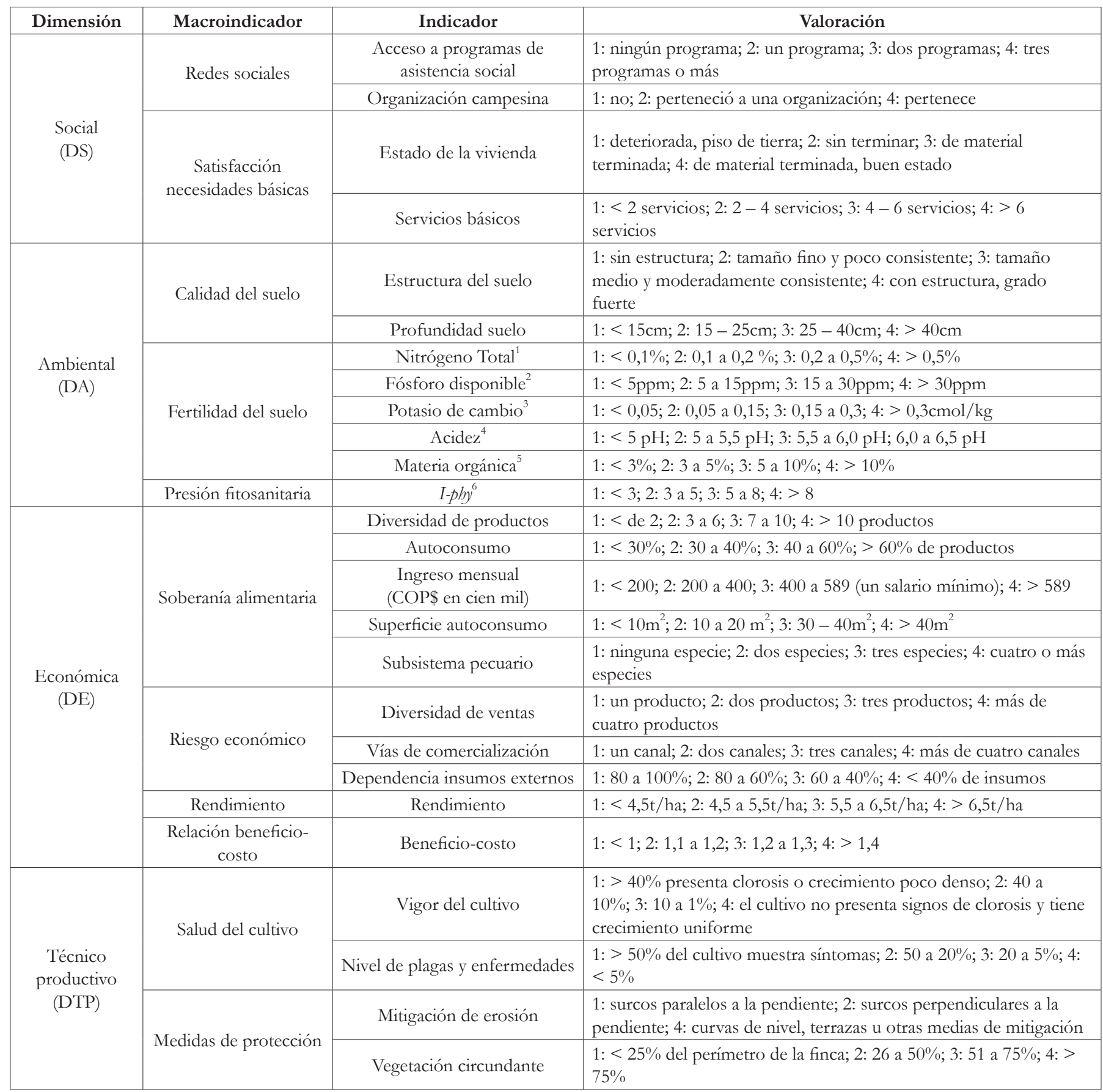

${ }^{1}$ Kjeldahl; ${ }^{2}$ Bray II y Kurtz; ${ }^{3}$ Acetato de amonio; ${ }^{4}$ Potenciómetro; ${ }^{5}$ Walkley-Black; ${ }^{6}$ Valores obtenidos de Álvarez et al. (2020). 
Análisis y presentación de resultados. Se desarrolló el método AMOEBA, para representar los macroindicadores evaluados y determinar, en este gráfico, los Puntos Críticos de Sustentabilidad (PCS), cuando el valor calculado era menor a 0,65. Además, se calculó el Índice General de Sustentabilidad (IGS), al establecer el promedio entre la dimensión social (DS), ambiental (DA), económica (DE) y técnico-productiva (DTP).

\section{RESULTADOS Y DISCUSIÓN}

Estimación de indicadores, primera fase. La evaluación en terreno demostró que la participación de los productores de arveja en organizaciones asociativas y acceso a programas de asistencia rural para los clústeres $\mathrm{C} 2$ y C3, en la mayoría de los casos, tuvo un valor superior a 2,0 puntos, en la escala. En promedio, dos integrantes por familia están vinculados a grupos de comercialización, asistencia técnica o proyectos productivos; además, se identificó apoyo institucional, especialmente, relacionado con la alcaldía municipal (Tabla 1).

Conviene señalar que el cultivo de arveja, desde finales de los años 90, cuando fue establecido en la región como una alternativa para sustituir la siembra tradicional de trigo, contó con programas apoyados por instituciones públicas y privadas, para que se consolidara dicha reconversión, usando como estrategia, la conformación de asociaciones campesinas, lo cual, hace que, actualmente, se identifique esta característica en el sistema productivo.

Tabla 1. Evaluación de los indicadores de sustentabilidad de fincas productoras de arveja en Ipiales-Nariño.

\begin{tabular}{|c|c|c|c|c|}
\hline \multirow{2}{*}{ Dimensión } & \multirow{2}{*}{ Indicador } & \multicolumn{3}{|c|}{ Tipo de finca evaluada (clúster) } \\
\hline & & $\mathrm{C} 1$ & $\mathrm{C} 2$ & C3 \\
\hline \multirow{4}{*}{$\begin{array}{l}\text { Social } \\
\text { (DS) }\end{array}$} & $\begin{array}{l}\text { Acceso a programas de asisten- } \\
\text { cia social }\end{array}$ & 1,1 & 2,2 & 2,4 \\
\hline & Organización campesina & 1,5 & 3,2 & 2,7 \\
\hline & Estado de la vivienda & 3,7 & 3,1 & 2,7 \\
\hline & Servicios básicos & 3,7 & 2,9 & 2,5 \\
\hline \multirow{8}{*}{$\begin{array}{c}\text { Ambiental } \\
\text { (DA) }\end{array}$} & Estructura del suelo & 2,9 & 3,9 & 3,6 \\
\hline & Profundidad suelo & 2,7 & 3,9 & 3,4 \\
\hline & Nitrógeno total & 2 & 2,5 & 2,6 \\
\hline & Fósforo disponible & 1,8 & 2,1 & 2,1 \\
\hline & Potasio de cambio & 3,8 & 4,0 & 3,9 \\
\hline & Acidez & 1,7 & 1,5 & 1,5 \\
\hline & Materia orgánica & 2,4 & 3,0 & 3,0 \\
\hline & $I-P b y$ & 2,6 & 1,8 & 1,9 \\
\hline \multirow{10}{*}{$\begin{array}{c}\text { Económica } \\
\text { (DE) }\end{array}$} & Diversidad de productos & 1,8 & 2,4 & 3,3 \\
\hline & Autoconsumo & 1,5 & 2,4 & 3,1 \\
\hline & Ingreso mensual & 3,9 & 2,2 & 1,7 \\
\hline & Superficie autoconsumo & 1,3 & 3,1 & 3,2 \\
\hline & Subsistema pecuario & 1,4 & 2,3 & 2,6 \\
\hline & Diversidad de ventas & 1,4 & 2,7 & 2,0 \\
\hline & Vías de comercialización & 1,4 & 1,1 & 1,3 \\
\hline & Dependencia insumos externos & 1,0 & 1,4 & 1,3 \\
\hline & Rendimiento (t/ha) & 3,8 & 3,6 & 3,0 \\
\hline & Beneficio-costo & 3,1 & 3,6 & 3,0 \\
\hline \multirow{4}{*}{$\begin{array}{l}\text { Técnico produc- } \\
\text { tivo }\end{array}$} & Vigor del cultivo & 3,8 & 2,9 & 2,7 \\
\hline & Nivel de plagas y enfermedades & 3,8 & 3,1 & 2,6 \\
\hline & Mitigación de erosión & 1,8 & 3,2 & 1,9 \\
\hline & Vegetación circundante & 2,2 & 3,0 & 3,0 \\
\hline
\end{tabular}


Por otro lado, la satisfacción de necesidades básicas fue especialmente baja para estos clústeres, en donde, de diez servicios básicos identificados en la región, se contabilizaron en estas fincas un promedio de cuatro, siendo crítico el limitado acceso de agua potable, salud pública, la ausencia de alcantarillado y red de transporte.

En contraste, los campesinos agrupados en C1, como se muestra en la tabla 1, presentaron menor interés en la participación de organizaciones o búsqueda de apoyo institucional y exhibieron condiciones más favorables, comparativamente, en los indicadores vivienda y servicios básicos, sin llegar a tener la totalidad de necesidades cubiertas.

En torno a la dimensión ambiental, estudios antecedentes demostraron que, en la región, el cultivo de arveja sigue un patrón generalizado de alto uso de insumos sintéticos, especialmente plaguicidas y las prácticas sobre el suelo, sugieren un deterioro de la fertilidad natural (Álvarez et al. 2019); por tanto, los indicadores usados buscaron describir aspectos vinculados con la cadena de acción -efectos-, como sugiere Latruffe et al. (2017), determinados a partir de las características fisicoquímicas del suelo y del índice de presión fitosanitaria I-Phy.

Se determinó que los indicadores en C1 presentaron un valor inferior con relación a las formas menos intensivas de producción, que no usaron tractor para preparar la parcela, identificando una estructura poco definida y profundidad efectiva, entre 15 a $20 \mathrm{~cm}$. Entretanto, C2 y C3 mostraron, en la mayor parte de las fincas, una estructura consistente, agregados definidos y una profundidad promedio de 25 a $40 \mathrm{~cm}$ (Tabla 1).

Ante esto, Gliessman (2002) identificó a la mecanización intensiva, como un punto que limita la sustentabilidad del sistema; para este autor, prevalece en el modelo productivo actual, la necesidad de generar un crecimiento rápido de las raíces, facilitar la siembra, controlar las arvenses, entre otros argumentos inmediatos, antes de reflexionar sobre el futuro del suelo y su degradación.

Los valores generales de acidez $(\mathrm{pH}=5,0)$ y fósforo disponible $(\mathrm{P}=$ 9,3 ppm) fueron limitantes para la producción en todos los clústeres. Debido al material parental de la zona, el potasio de cambio, con un promedio de $0,48 \mathrm{cmol} / \mathrm{kg}$, presentó una alta disponibilidad, mostrando un comportamiento independiente (Tabla 1).

En este sentido fue importante contar con una tipificación previa, ya que se demostró que los pequeños campesinos tuvieron una mejor valoración en los indicadores de la dimensión ambiental (Tabla 1). Estos resultados permiten entender la necesidad y la vigencia de estas formas de producción, entre otras razones, porque ocupan y aprovechan, de mejor manera, los ecosistemas vulnerables (Vélez, 2015).

Numerosas son las investigaciones que concluyen que la sustentabilidad inicia con el incremento de la fertilidad, siguiendo un enfoque sistémico, que lleve a que un equilibrio bajo el suelo, se manifieste arriba y viceversa. Esto, a su vez, mejora las relaciones que el suelo promueve, otorgando a los campesinos y a sus familias autonomía, resiliencia y productividad a largo plazo que, en este caso, se irá reduciendo en las fincas de C1, de seguir el modelo actual.

Se confirmó también, la dependencia generalizada en el uso de plaguicidas para el control de arvenses, microorganismos e insectos plaga del cultivo (Tabla 1). De acuerdo con el estudio de Álvarez et al. (2020), la afectación al agua subterránea, agua superficial, aire y riesgo al consumidor, se incrementa en los sistemas más vulnerables, asociados a C2 y C3, debido a que la elección de los productos plaguicidas se basa en el costo y se ignora el grado de toxicidad que estos pueden tener y que interactúan con las condiciones agroambientales de la región, acrecentando el riesgo, presentando un puntaje menor en el indicador I-phy.

La diferencia entre indicadores más considerable, se presentó en la dimensión económica, como se observa en la tabla 1, ligada a la generación local de ingresos, que estuvo por debajo del umbral, mostrando que, en promedio, la entrada económica familiar fue menor a un salario mínimo. Aun así, el cultivo de arveja, en los últimos años, ha sido el único que ofrece una relativa estabilidad de precio y demanda continua en el mercado.

Paralelamente, en los grupos de campesinos con menor área dedicada al cultivo de arveja (C2 y C3), a partir de la "chagra", se reportó el mayor puntaje en los indicadores diversidad de productos, superficie de autoconsumo, porcentaje de autoconsumo, subsistema pecuario y diversidad de ventas (Tabla 1). En la chagra, se registró un promedio de 11 productos vegetales y dos especies animales por finca, que confieren alimentos arraigados culturalmente y producidos con reducido uso de plaguicidas, fertilizantes sintéticos o concentrados animales, generando un mayor grado de soberanía alimentaria para la familia.

En el grupo C1, el indicador ingreso mensual fue mayor; la economía interna dependió exclusivamente del cultivo de arveja (Tabla 1). Estas características sugieren una mayor especialización y homogenización del proceso productivo con mayores costos que, de acuerdo con Vélez (2015), trae consigo la monetización de la economía familiar, repercutiendo sobre la vulnerabilidad de la finca, debido a la reducción de su flexibilidad productiva, que constituye una estrategia de adaptabilidad y de persistencia del campesino.

La adquisición de los productos alimentarios en C1, se sustentó en el mercado, evidenciando el cambio de modo de vida y los valores rurales, pues aun reconociendo el beneficio de la diversificación, este grupo destinó la totalidad del área de la finca y tiempo de trabajo al cultivo que consideran principal. Esta característica muestra el deterioro del conocimiento, el "saber-hacer" y al mismo tiempo afecta el sistema alimentario local, al promover la intensificación de un modelo simplificado.

Para Latruffe et al. (2017), el riesgo de inestabilidad económica se incrementa cuando las familias, además, no diversifican su economía y se crea dependencia a pocos canales de comercialización. Describiendo la situación general del macro indicador riesgo 
económico, independientemente del clúster valorado, conflicto que deberá ser abordado por la comunidad.

La dimensión técnico-productiva, por su parte, reforzó los resultados que demostraron alta dependencia de insumos externos en la producción de arveja. Los indicadores que midieron el vigor y la sanidad del cultivo (nivel de plagas y enfermedades), revelaron un comportamiento aceptable cuando el campesino aplicó el máximo nivel de plaguicidas y de fertilización, pero fue sensible ante la reducción en el uso de estos productos, demostrando que, actualmente, la capacidad del sistema para autorregularse es baja (Tabla 1).

Numerosos son los casos, en los cuales, la dependencia de estos productos comúnmente termina abatiendo los mecanismos de regulación ecológica, con ello, por más que se incremente la aplicación, los rendimientos decrecen (Paleologos et al. 2017; diseño y manejo de agroecosistemas sustentables. Uno de los mayores desafíos en la actualidad, es lograr disminuir o eliminar el uso de insumos (caros y peligrososRosset \& Altieri, 2018). Si se tiene en cuenta que la oferta de "paquetes tecnológicos" en el sur de Nariño elevó el rendimiento potencial de 3,4t/ha a 8t/ha, en solo una década (MADR, 2018), se debe reflexionar sobre el nivel de extracción y el impacto que el sistema puede soportar a mediano y largo plazo.

Se suma a esto, la ausencia generalizada de estrategias de conservación del suelo como curvas de nivel, terrazas, coberturas, barreras vivas, entre otras, con lo cual, se determinó que el productor no considera la topografía para establecer el cultivo, incrementando el riesgo de pérdida de la fertilidad en aquellas fincas con topografía quebrada, como el caso de C1 y C3 (Tabla 1).

Finalmente, el indicador vegetación circundante mostró que las fincas que cuentan con arbustos o árboles integrando al cultivo de arveja (51 al 75\% del perímetro), mejoraron la calificación de vigor del cultivo y rendimiento, en comparación con aquellas con ausencia de esta estructura (Tabla 1), por lo cual, se sugiere una evaluación específica, por la importancia que puede tener para la región el implementar esta estrategia, a nivel del paisaje, como una respuesta ante la variabilidad y el cambio climático, cada vez más inminente.

Estimación de indicadores, segunda fase. Los indicadores correspondientes a esta fase de evaluación enfrentaron dos lógicas: la de rentabilidad a corto plazo con mayor presión de los recursos naturales, versus sistemas más diversos, más adaptados, pero menos escalables, bajo la racionalidad de la economía tradicional, generando la necesidad de reflexionar para la región, una nueva forma de producción de arveja, económicamente viable, pero responsable y sensible, con las relaciones sociales, ambientales y técnico-productivas, que se establece en la finca.

El análisis de costos estableció que el cultivo requiere una inversión entre \$9.882.000 y \$11.346.016 pesos por hectárea (junio 2017), con pequeñas variaciones entre tipos de campesinos, cuando se extrapola a una unidad de área común. El clúster C1 presentó un rendimiento de $6,82 \mathrm{t} / \mathrm{ha}$, con una relación costo beneficio de $31 \%$; por su parte, el clúster C2 y C3 presentaron un rendimiento menor con 6,22 y $5,94 \mathrm{t} / \mathrm{ha}$, respectivamente, así como una relación costo beneficio del 34 y $27 \%$, confirmando que el primer clúster, por la suma de características, representa un tipo de agricultura más intensivo, en donde la producción prima frente al deterioro y empobrecimiento de los componentes de la finca.

Este potencial de cosecha sumado a un requerimiento sostenido en el mercado y políticas agropecuarias locales, que promueven la siembra de arveja, permite conocer las lógicas, por las cuales, los campesinos aceptan y expanden el cultivo, asumiendo las diferentes externalidades, especialmente, sobre el ambiente.

Como un punto importante, se pudo establecer que, en todos los casos, la relación de retorno fue positiva, acercándose o superando al criterio de desempeño para sistemas campesinos en BoyacáColombia, sugerido por Fonseca et al. (2016), en donde el 30\% de utilidad es favorable.

En los tres clústeres, la dimensión económica tuvo un potencial razonable, pero no puede, ni debe ser el único factor de calificación, lo cual, conduce a la necesidad de una reorganización integral, cuyo objetivo permita crear un nuevo equilibrio en las fincas dedicadas al cultivo de arveja, buscando que se presenten condiciones adecuadas en las demás dimensiones.

Puntos Críticos de Sustentabilidad (PCS). Cada tipo de clúster, al tener situaciones particulares, presentó puntos críticos específicos, generados por las diferentes relaciones que se establecen a nivel de cultivo, finca y región, de forma sistémica. En este sentido, los participantes del estudio confirmaron los problemas resultantes del modelo productivo adoptado, que registró, al menos, un PCS, configurando un conflicto generalizado en el sur de Nariño y suscitando dudas sobre la capacidad que tienen las fincas para soportar tal nivel de extracción, a largo plazo.

Con respecto al primer clúster, en la figura 1, se identificaron cuatro atributos, con valores inferiores al umbral crítico. El primero, relacionado con la individualidad asumida por los campesinos entorno a la interacción social e institucional, que incrementa la vulnerabilidad de las fincas, que enfrentan aisladamente los riesgos de la producción.

La capacidad de organización y de gestión de redes sociales, como los lazos de vecindad, grupos de comercialización, asociaciones campesinas, proyectos productivos, entre otros ejemplos, intensifican los mecanismos de reciprocidad y de cooperación y sirven de respaldo en momentos de crisis. Bravo et al. (2017) indican, además, que las fincas sustentables consideran la relación de la familiacomunidad, permitiendo desarrollar actividades como intercambio de semillas, de insumos, bienes o servicios, que hacen parte del proceso de producción dinamizado por las redes locales.

Los campesinos con características de C1 deberán considerar, igualmente, el acercamiento a las instituciones, que permita el 
Figura 1. AMOEBA, para determinar Puntos Críticos de Sustentabilidad, en los tres tipos de fincas productoras de arveja.

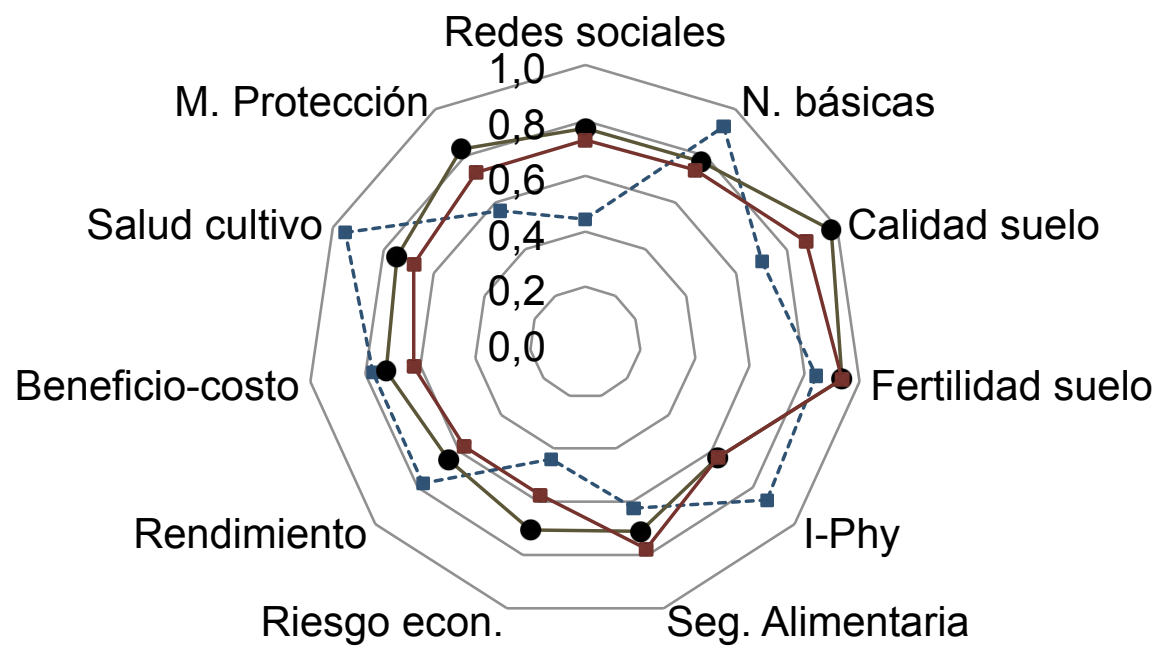

------ Clúster $1 \longrightarrow$ Clúster $2 \longrightarrow$ Clúster 3

fortalecimiento sociopolítico como estrategia agroecológica de sustentabilidad, bajo la salvedad, que las instituciones tienen la capacidad de influir adecuadamente en el territorio, cuando una comunidad está organizada y posee un convencimiento sobre la estrategia productiva conveniente.

El riesgo económico y la soberanía alimentaria, también se identificaron en este grupo, como PCS, que describen una baja diversidad de productos para autoconsumo y comercialización, confirmando la simplificación que trajo consigo el cultivo de arveja, en donde la agroecología, por el contrario, busca una continua adaptación a circunstancias cambiantes, que no permite tal nivel de especialización. Esta diversidad, además, opera como amortiguador para evitar el fracaso del sistema ante las fluctuaciones, incrementando la capacidad compensatoria o de resiliencia, si cae o falla una especie, otras pueden asumir su papel (Rosset \& Altieri, 2018).

El último PCS identificado en C1 estuvo relacionado con la presión en el uso del suelo, a través de la intensificación del sistema, especialmente, en los lotes que los productores tomaron en arrendamiento. Para Sajadian et al. (2017), la tenencia de tierra facilita la implementación de estrategias de conservación; para los arrendatarios o socios esta preocupación tiende a ser secundaria, sobresaliendo la necesidad inmediata por recuperar la inversión, muestra de ello, fue el hecho que, en ningún caso, a un lote tomado en arriendo se le realizó un manejo apropiado y el atributo de fertilidad del suelo fue el más bajo.

Por su parte, las fincas agrupadas en C2 presentaron el comportamiento más favorable, a partir de la estimación de los indicadores y los macroindicadores evaluados (Figura 1). Sobresale, en este caso, el uso extremado de productos plaguicidas con alta toxicidad, representado en el valor de $I-P h y$, aludiendo medidas de manejo urgentes, debido al riesgo potencial de contaminación.

Bajo este contexto, mientras un solo cultivo se mantenga en la finca como la base estructural, los problemas fitosanitarios seguirán siendo el resultado de un círculo vicioso, que perpetúe la aplicación de plaguicidas. Los sistemas simplificados limitan la posibilidad de autorregulación poblacional y expresión de las dinámicas de compensación; además, es cada vez más recurrente la pérdida de eficacia de estos productos, a causa de plagas tolerantes o resistentes (Gliessman, 2002; Nicholls et al. 2015).

La reflexión para este clúster debe iniciarse con el cambio de concepción en las prácticas de cultivo, permitiendo, paulatinamente, integrar estrategias de transición agroecológica adaptada al contexto, citando como ejemplo, el aumento de la eficiencia en la utilización de insumos, mediante el manejo integrado de plagas (MIP), la sustitución de insumos y, finalmente, el rediseño del sistema, estrategias que pueden mejorar el grado de sustentabilidad mostrado (Nicholls et al. 2015; Le Bellec et al. 2017; Álvarez et al. 2020).

Finalmente, los sistemas asociados a C3 presentaron cuatro PCS, (Figura 1), de los que fue concluido nuevamente el problema del uso de plaguicidas, que describe el indicador I-Phy, donde se proponen las consideraciones descritas en el anterior clúster, con que comparten este punto crítico.

Esta dependencia de plaguicidas redujo en este grupo la autosuficiencia de la finca, afectando en C3, la estabilidad económica de la familia, reportando un valor por debajo del umbral, en el atributo riesgo económico. Es necesario, por tanto, reducir los 
rendimientos decrecientes sin la necesidad de añadir cantidades crecientes de energía y nutrientes (Nicholls et al. 2015; Rosset \& Altieri, 2018), siendo contrario a lo evidenciado en campo, en donde fueron más numerosas y severas las externalidades vinculadas al uso de un paquete tecnológico cerrado, bajo las condiciones de este tipo de campesinos más vulnerables.

Fue paradójico que, a pesar de la elevada carga de plaguicidas aplicados, se obtuvieran, en estas condiciones, los valores más bajos en el atributo salud del cultivo, especialmente, cuando los sistemas asociados al clúster no contaron con una estructura vegetal acompañante o cuando la pendiente del terreno fue muy fuerte. La convivencia y la adaptación de la agricultura a la capacidad de soporte del medio deben primar en ambientes marginales, dado que el potencial de la finca tiende a disminuir rápidamente cuando se asume un uso inadecuado.

Para este clúster, el último PCS estuvo relacionado con el bienestar de los campesinos y sus familias, detectado por el atributo necesidades básicas (Figura 1), alertando sobre la necesidad de mayores esfuerzos en las políticas agrarias, representadas en los programas y planes gubernamentales, que inciden sobre la pequeña agricultura de la región. Para exigir los derechos como campesinos y cambiar políticas públicas, necesariamente, se precisa de una capacidad de ordenamiento y unas organizaciones fuertes, que contribuyan al progreso local, de la mano con el Estado. No en vano, los movimientos sociales son el medio de cultivo, sobre el cual, crece la agroecología y que ha generado, históricamente a nivel mundial, transformaciones profundas sobre la inequidad en torno a las necesidades básicas y el buen vivir.

Índice General de Sustentabilidad (IGS). El valor para este índice agregado, que fue calculado al establecer el promedio entre las cuatro dimensiones abordadas, social (DS), ambiental (DA), económica (DE) y técnico-productiva (DTP), ordenó el puntaje de mayor a menor en las fincas C2 [2,9= $(2,85+2,77+2,94+3,02) / 4], \mathrm{C} 1[2,65=$ $(2,50+2,58+2,59+2,93) / 4]$ y C3 $[2,62=(2,58+2,64+2,60+2,65) / 4]$, respectivamente. Si bien los tres clústeres superaron el valor medio de la escala, definido como el límite de una condición discreta de sustentabilidad, dista de una situación adecuada a largo plazo.

Se debe aclarar que este tipo de evaluaciones presentan un concepto dinámico, ya que las condiciones o aptitudes de un sistema pueden cambiar en el tiempo; sin embargo, la utilidad de identificar y de entender las problemáticas bajo una visión holística y sistémica, permiten avanzar a un nivel superior de sustentabilidad, como describe Bravo et al. (2017); además, al atender los puntos críticos dentro de cada dimensión y reconocer las experiencias de las fincas con puntajes altos de IGS, se pueden identificar referentes, que permitan proponer ajustes en el sistema.

Una finca sustentable es una alternativa viable, que les permite a las familias campesinas desarrollarse y fortalecerse, de tal manera, que puedan revalorar la fuerza de trabajo familiar o convertirse en generadoras de empleo justo, constituirse en sistemas que combinen tradiciones y tecnologías, a través del uso responsable del medio y en donde se establezca una relación de carácter recíproco, reduciendo la presión generada por la agricultura convencional.

Como se ha expuesto a lo largo de esta investigación, las condiciones particulares de los tres clústeres evaluados, sugieren medidas de monitoreo y de atención, que puedan ser resueltas, mediante estrategias endógenas, adaptadas al contexto sociocultural e histórico de la subregión sur de Nariño, que pueden trascender a planos técnicos y políticos más amplios, incrementando la sustentabilidad, como propiedad emergente.

Conflictos de intereses: El manuscrito fue preparado y revisado con la participación de todos los autores, quienes declaramos que no existe ningún conflicto de intereses que ponga en riesgo la validez de los resultados presentados.

\section{REFERENCIAS}

1. ÁlvareZ, D.; CHAVES, D.; GÓMEZ, E.; HURTADO, A. 2020. Estimación del riesgo ambiental causado por plaguicidas en cultivos de arveja de Ipiales, NariñoColombia. TecnoLógicas (Colombia). 23(47):77-91. https:/ / doi.org//10.22430/22565337.1404

2. ÁLVAREZ, D.; GÓMEZ, E.; ORDÓÑEZ, H. 2019. Tipología de fincas productoras de arveja (Pisum sativum L.) en la subregión Sur de Nariño, Colombia. Ciencia y Tecnología Agropecuaria (Colombia). 20(3):659-677. https://doi. org/10.21930/rcta.vol20_num3_art:1593

3. BLANDI, M.; SARANDÓN, S.; FLORES, C.; VEIGA, I. 2015. Evaluación de la sustentabilidad de la incorporación del cultivo bajo cubierta en la horticultura platense. Rev. Fac. Agr. (Argentina). 114(2):51-264.

4. BRAVO, C.; MARÍN, H.; MARRERO, P.; RUIZ, M.; TORRES, B.; NAVARRETE, H.; DURAZNO, G.; CHANGOLUISA, D. 2017. Evaluación de la sustentabilidad mediante indicadores en unidades de producción de la provincia de Napo, Amazonia Ecuatoriana. Bioagro (Venezuela). 29(1):23-36.

5. FONSECA, J.; CLEVES, J.; LEÓN, T. 2016. Evaluación de la sustentabilidad de agroecosistemas familiares campesinos en la microcuenca del río Cormechoque (Boyacá). Rev. Ciencia y Agricultura (Colombia). 13(1):29-47.

6. GLIESSMAN, S. 2002. Agroecología: Procesos ecológicos en agricultura sostenible. Ed. CATIE (Costa Rica). 359p.

7. LATRUFFE, L.; DIAZABAKANA, A.; BOCKSTALLER, C.; DESJEUX, Y.; FINN, J.; KELLY, E.; RYAN, M.; UTHES, S. 2017. Measurement of sustainability in agriculture: A review of indicators. Studies in Agricultural Economics (Hungría). 118(3):123-130. http://dx.doi.org/10.7896/j.1624 
8. LE BELLEC, F.; SCORBIAC, M.; SAUZIER, J. 2017. Les pratiques phytosanitaires des producteurs de légumes de l'île Maurice: Impacts et perspectives de changement. Cahiers Agricultures (Francia). 26(55):1-9. https://doi.org/10.1051/ cagri/2017038

9. MINISTERIO DE AGRICULTURA Y DESARROLLO RURAL, MADR. 2018. Área cosechada, producción y rendimiento de arveja, 1986-2017. Disponible desde Internet en: https://goo.gl/jHL4HK (con acceso 06/09/2019).

10. NICHOLLS, C.; ALTIERI, M.; VÁZQUEZ, L. 2015. Agroecología: Principios para la conversión y el rediseño de sistemas agrícolas. Agroecología (España). 10(1):61-72.

11. PAlEOlOGOS, M.; IERMANÓ, M.; BLANDI, M.; SARANDÓN, S. 2017. Las relaciones ecológicas: un aspecto central en el rediseño de agroecosistemas sustentables, a partir de la Agroecología. Rev. do desenvolvimento regional
(Brasil). 22(2):92-115. https://doi.org/10.17058/redes. v22i2.9346

12. ROSSET, P.; ALTIERI, M. 2018. Agroecología ciencia y política. Ed. SOCLA (Bolivia). 206p.

13. SAJADIAN, M.; KHOSHBAKHT, K.; LIAGHATI, H.; VEISI, H.; MAHDAVI, A. 2017. Developing and quantifying indicators of organic farming using analytic hierarchy process. Ecological Indicators (Países Bajos). 83:103-111. https://doi.org/10.1016/j.ecolind.2017.07.047

14. SARANDÓN, S.; FLORES, C. 2009. Evaluación de la sustentabilidad en agroecosistemas: Una propuesta metodológica. Agroecología (España). 4(1):19-28.

15. VÉLEZ, L. 2015. Adaptabilidad y persistencia de las formas de producción campesinas. Ed. Universidad Nacional de Colombia (Colombia). 112p. 